DESPLAZAMIENTO FORZADO INTERNO COMO CONSECUENCIA DE UNA INUNDACIÓN EN LA ENTIDAD DE TABASCO, MÉXICO.

\title{
DESPLAZAMIENTO FORZADO INTERNO COMO CONSECUENCIA DE UNA INUNDACIÓN EN LA ENTIDAD DE TABASCO, MÉXICO
}

\section{INTERNAL FORCED DISPLACEMENT AS A CONSEQUENCE OF A FLOOD IN THE ENTITY OF TABASCO, MEXICO}

\author{
Mariam Gisela Ruíz Ramón*
}

* Licenciada en Derecho. División Académica de Ciencias Sociales y Humanidades de la Universidad Juárez Autónoma de Tabasco. ORCID: https://orcid.org/0000-0003-4587-7730.

Dirección para correspondencia: investigacionesdocentes@hotmail.com 
DESPLAZAMIENTO FORZADO INTERNO COMO CONSECUENCIA DE UNA INUNDACIÓN EN LA ENTIDAD DE TABASCO, MÉXICO.

\section{RESUMEN}

OBJETIVO: Analizar las consecuencias del desplazamiento forzado interno originado por causas ambientales, en específico como consecuencia de una inundación en la entidad de Tabasco, México.

MATERIAL Y MÉTODO: Se llevó a cabo mediante técnicas como la observación, entrevistas no estructuras y aplicación de 200 cuestionarios de 44 reactivos, posteriormente el análisis de la información y finalmente elaboración de base de datos e informe final.

RESULTADOS: El objeto de estudio, en este caso los habitantes desplazados señalaron o manifestaron que como consecuencia del desplazamiento forzado interno tuvieron procesos complejos de adaptación, afectaciones a sus derechos humanos, además de estar y sentirse en estado de vulnerabilidad y riesgo por el poco o nulo tratamiento de los desechos en el fraccionamiento.

CONCLUSIONES: El desplazamiento forzado interno sin medidas de seguridad, protocolos de actuación y sin considerar las necesidades básicas humanas, sólo cumplió un compromiso en cuanto a infraestructura, sin prever consecuencias de adaptación, garantía y acceso a los derechos de vida digna, seguridad humana, vivienda, agua, alimentación, salud.

PALABRAS CLAVE: Refugiados. Adaptabilidad. Reubicados. Desplazamiento. Medio ambiente.

\section{ABSTRACT}

OBJECTIVE: To analyze the consequences of the internal forced displacement caused by environmental causes, specifically as a consequence of a flood in the state of Tabasco, Mexico.

MATERIAL Y METHOD: It was carried out using techniques such as observation, unstructured interviews and the application of 200 questionnaires of 44 items, subsequently the analysis of the information and finally the elaboration of the database and final report. 
DESPLAZAMIENTO FORZADO INTERNO COMO CONSECUENCIA DE UNA INUNDACIÓN EN LA ENTIDAD DE TABASCO, MÉXICO.

RESULTS: The object of study, in this case the displaced inhabitants stated that as a consequence of the internal forced displacement, they had complex adaptation processes, violations of their human rights, in addition to being and feeling in a state of vulnerability and risk due to the little or no treatment of waste in their neighborhood.

CONCLUSIONS: The internal forced displacement without security measures, action protocols and without considering basic human needs, only fulfilled a commitment in terms of infrastructure, without foreseeing consequences of adaptation, guarantee and access to the rights to a dignified life, human security, housing, water, food, health.

KEY WORDS: Refugees. Adaptability. Relocated. Displacement. Environment.

\section{INTRODUCCIÓN}

En México, de acuerdo a la Comisión Mexicana de Defensa y Protección de Derechos Humanos durante el periodo del año 2009 a enero del año 2017, en un aproximado de 310,527 personas tuvieron que desplazarse de manera interna debido a la violencia, conflictos territoriales, religiosos o políticos de forma directa siendo estos los de mayor tendencia.

En el año 2016, se registraron al menos 29 episodios de desplazamiento masivo en el país, impactando a 23,169 personas en 12 entidades, como son: Chiapas, Chihuahua, Durango, Guerrero, Hidalgo, Jalisco, Michoacán, Oaxaca, Sinaloa, Tamaulipas, Veracruz y Zacatecas; las cuales sin duda, ha ido agudizando la situación y emergiendo otros Estados en igual o peor circunstancias.

La población que regularmente se encuentra en un estado de vulnerabilidad y riesgo ante esta situación, son las mujeres, niñas, niños, población indígena, campesinos, activistas (grupos vulnerables). A su vez, como problema social agudiza el acceso y garantía de los derechos a la vida, integridad personal, libertad y seguridad personal, alimentación, libre circulación y residencia, garantías procesales y protección judicial, vida familiar y cultural digna, salud, educación, vivienda digna y adecuada, empleo, propiedad privada, a la vida privada y el domicilio. 
DESPLAZAMIENTO FORZADO INTERNO COMO CONSECUENCIA DE UNA INUNDACIÓN EN LA ENTIDAD DE TABASCO, MÉXICO.

En la entidad de Tabasco, el desplazamiento interno lo manejaron y aplicaron con la categoría de reubicados, tanto el gobierno y la población, está última identificándose como tal. El proceso de "reubicación" se efectuó con solo otorgar una vivienda "propia"; sin embargo, no solo era construir casas, sino, de forma integral crear las viviendas conforme a las condiciones mínimas que establece el acceso a una vivienda digna.

\section{MARCO TEÓRICO}

De acuerdo a la agencia de la ONU para los refugiados ACNUR, se entiende por desplazados internos aquellos individuos o grupos de personas que han sido forzados a huir de sus hogares para escapar del conflicto armado, la violencia generalizada, los abusos de los derechos humanos o los desastres naturales o provocados por el ser humano. Los refugiados y desplazados internos con frecuencia abandonan sus hogares por las mismas razones; sin embargo, se distinguen de la siguiente manera: los civiles son reconocidos internacionalmente como refugiados cuando cruzan la frontera nacional en busca de asilo en otro país.

Los desplazados internos, mejor conocidos como "IDP", por el acrónimo inglés de "Internally Displaced People", permanecen, por cualesquiera razones que sean, en sus propios países. En el caso de adaptabilidad para el presente trabajo se entenderá como la capacidad o habilidad de un grupo social de ajustarse a cambios ambientales con fines de supervivencia y sustentabilidad (Quintero, 2005, p. 14).

De acuerdo a la ONU, a través de los Principios rectores (Naciones Unidas, 1998), se entiende por Desplazados Internos a todas aquellas:

"Personas o grupos de personas que se han visto forzadas u obligadas a escapar o huir de su hogar o de su lugar de residencia habitual, en particular como resultado o para evitar los efectos de un conflicto armado, de situaciones de violencia generalizada, de violaciones de los derechos humanos o de catástrofes naturales o provocadas por el ser humano, y que no han cruzado una frontera estatal internacionalmente reconocida".

El Desplazamiento Forzado Interno (DFI) también es considerada una violación a los derechos humanos, que se presenta cuando personas o grupos de personas huyen de su hogar o lugar de residencia habitual hacia otra colonia de su mismo municipio, hacia otro municipio de su Estado o hacia otro Estado del país, para evitar o después de haber sido víctimas de una situación de violencia generalizada, de un conflicto armado, de violaciones a los derechos humanos o de RUÍZ-RAMÓN M. G.

SEPTIEMBRE-DICIEMBRE 2020. Año 26, Número 76. Págs. 338-351 
DESPLAZAMIENTO FORZADO INTERNO COMO CONSECUENCIA DE UNA INUNDACIÓN EN LA ENTIDAD DE TABASCO, MÉXICO.

catástrofes naturales o provocadas por el ser humano (CMDPDH, 2019), se comprende que la manifestación del desplazamiento se presenta a través de un colectivo de personas por motivos diversos, que en caso de no desplazarse las consecuencias pueden resultar violentas.

El documento de Los Principios Rectores sobre el Desplazamiento Interno ofrecen un patrón para determinar en qué momento el desplazamiento (no sólo el interno) se convierte en una cuestión de derechos humanos de interés nacional e internacional, incluso por motivos relacionados con el daño medioambiental; describe los parámetros del derecho a no ser desplazado de forma arbitraria e incluyen las garantías que deben tenerse en cuenta en el caso de que el desplazamiento sea inevitable, a fin de reducir sus efectos al máximo, así como las obligaciones concretas que tienen los Estados de proteger contra el desplazamiento a los grupos con una dependencia especial o apego particular a sus tierras.

María Stavropoulou (2008) señala, que en esos casos, las personas implicadas no son sólo las víctimas de desastres naturales, sino también los desplazados arbitrariamente, ya sean internos o refugiados. Definirlos como 'refugiados medioambientales' o 'refugiados debido al cambio climático no sirve más que para ampliar la difusión de la cuestión. Por lo anterior, es convenientes citar principio 7 de los Principios Rectores sobre el Desplazamiento Interno que a la letra dice:

1. Antes de decidir el desplazamiento de personas, las autoridades competentes se asegurarán de que se han explorado todas las alternativas viables para evitarlo. Cuando no quede ninguna alternativa, se tomarán todas las medidas necesarias para minimizar el desplazamiento y sus efectos adversos.

2. Las autoridades responsables del desplazamiento se asegurarán en la mayor medida posible de que se facilita alojamiento adecuado a las personas desplazadas, de que el desplazamiento se realiza en condiciones satisfactorias de seguridad, alimentación, salud e higiene y de que no se separa a los miembros de la misma familia.

3. Si el desplazamiento se produce en situaciones distintas de los estados de excepción debidos a conflictos armados y catástrofes, se respetarán las garantías siguientes:

(a) La autoridad estatal facultada por la ley para ordenar tales medidas adoptará una decisión específica.

(b) Se adoptarán medidas adecuadas para facilitar a los futuros desplazados información completa sobre las razones y procedimientos de su desplazamiento y, en su caso, sobre la indemnización y el reasentamiento;

(c) Se recabará el consentimiento libre e informado de los futuros desplazados;

(d) Las autoridades competentes tratarán de involucrar a las personas afectadas, en particular las mujeres, en la planificación y gestión de su reasentamiento;

(e) Las autoridades legales competentes aplicarán medidas destinadas a asegurar el cumplimiento de la ley cuando sea necesario; y

(f) Se respetará el derecho a un recurso eficaz, incluida la revisión de las decisiones por las autoridades judiciales competentes.

RUÍZ-RAMÓN M. G.

SEPTIEMBRE-DICIEMBRE 2020. Año 26, Número 76. Págs. 338-351 
DESPLAZAMIENTO FORZADO INTERNO COMO CONSECUENCIA DE UNA INUNDACIÓN EN LA ENTIDAD DE TABASCO, MÉXICO.

Aunado a lo anterior, es necesario mencionar como a nivel estatal, nacional e internacional aumenta el número de personas vulnerables que viven en tierras marginales expuestas al cambio climático. Para ello, es necesario que la aplicación de estrategias de adaptación, junto con la implementación de una buena gestión de riesgos de catástrofes, puede reducir, adecuar o manejar la vulnerabilidad frente al cambio climático, requiriendo que los diversos actores trabajen en distintos ámbitos, realicen un esfuerzo colectivo y traspasen los límites sectoriales.

Teniendo en mente, que si bien no todas las causas del DFI se relacionan con violaciones a los derechos humanos (Silva \& Martínez, 2019), como es el caso de los desastres naturales que no son el resultado de la intervención del ser humano; sin embargo, un desastre natural que pudo ser previsible y ante el cuál la autoridad omitió desplegar las acciones preventivas para la protección de la población, puede conducir a la probable responsabilidad del Estado por dichas omisiones; lo mismo si con posterioridad al desplazamiento, el Estado omitiera prestar la asistencia que requiera el caso. Por tanto, las violaciones a los derechos humanos en el DFI pueden presentarse también con posterioridad a un desastre natural (CNDH, 2016).

Es así, que se debe de comprender la indivisibilidad e interdependencia de los derechos humanos (Hernández, 2016) ante una situación de vulnerabilidad y riesgo colectivo en que el Estado pueda responder acorde las obligaciones internacionales en materia de derechos humanos (Jaimes, 2014), además de prever los principios de prevención y precautorio (Silva, 2019) en las políticas públicas ambientales.

\section{MATERIAL Y MÉTODO}

El trabajo de investigación: "Desplazamiento Forzado Interno Ambiental. Caso: Fraccionamiento 27 de Octubre". Es un trabajo basado en una metodología mixta que permitió comprender de forma cuantitativa (Briones, 2001) el porcentaje de personas y viviendas en condiciones de desplazamiento y cualitativamente el proceso de adaptabilidad, así como las formas de percepción de condición de vida, una vez desplazados. La estrategia para acercarnos a nuestro tema objeto de investigación, fue a través de herramientas y técnicas, como la observación, entrevistas no estructuras y aplicación de 200 cuestionarios de 44 reactivos (Garza, 2013), posteriormente el análisis de la información y finalmente elaboración de base de datos e informe final. 
DESPLAZAMIENTO FORZADO INTERNO COMO CONSECUENCIA DE UNA INUNDACIÓN EN LA ENTIDAD DE TABASCO, MÉXICO.

\section{RESULTADOS Y DISCUSIÓN}

\section{Fraccionamiento 27 de Octubre}

En Tabasco el desplazamiento interno por cuestiones de catástrofes ambientales (Prieur, 2009) tuvo presencia con las inundaciones suscitadas en el año 2007, cuando sectores de la población se desplazaron hacia otros Estados, existió un éxodo de la población no sólo rural sino urbana hacia refugios temporales y/o albergues.

Derivado de ello, durante esta situación una de las ventajas fue el fortalecimiento y activación de las redes familiares, vínculos de una residencia anterior, relaciones solidarias con las localidades vecinas o localidades cercanas de la región (Salazar, 2014). Sin embargo, después de este suceso el gobierno estatal y federal, decidieron implementar el desplazamiento interno de las familias que se encontraban en zona vulnerable (orilla de río o cuerpos de agua).

En el caso que nos ocupa las familias que habitaban en la colonia Asunción Castellanos, Tierra Colorada, Casa Blanca, Sector Compuerta, Indeco y la Manga I, fueron "reubicados"1 hacia el Fraccionamiento 27 de Octubre, en la cual se construyeron 880 casas.

Las familias que fueron desplazadas a este Fraccionamiento, se han ido adaptando a las siguientes circunstancias:

Nueva forma y estilo de vida.

Adaptar sus necesidades a la infraestructura de la casa.

- Crear espacios verdes y obtener sombra para sus viviendas o pequeños espacios para sembrar diversos alimentos.

Adaptarse a la escasez de mala calidad y/o ausencia de servicios básicos como agua, limpia y salud.

Adaptación a un nuevo tipo de organización e integración con vecinos.

Adaptarse a los servicios de educación y transporte; en algunos casos propicios a buscar nueva fuentes de empleo o emplearse con algún tipo de oficio.

Adaptarse a nuevas formas de vulnerabilidad y riesgo como el de la seguridad y como el de la acumulación de los desechos en los hogares propiciando que en tiempos de lluvia el agua quede estancada y tape los drenajes.

\footnotetext{
${ }^{1}$ El gobierno utilizó la categoría de reubicados para las familias que fueron desplazadas al Fraccionamiento 27 de Octubre; las familias y personas entrevistadas también se ubican con la categoría de reubicados.

RUÍZ-RAMÓN M. G.
}

SEPTIEMBRE-DICIEMBRE 2020. Año 26, Número 76. Págs. 338-351 
DESPLAZAMIENTO FORZADO INTERNO COMO CONSECUENCIA DE UNA INUNDACIÓN EN LA ENTIDAD DE TABASCO, MÉXICO.

Esto sin duda con lleva a considerar que para adaptarse, las familias requirieron de flexibilidad a su vez mantener y acceder a grados de flexibilidad acorde a como emergían o emergen circunstancias no previstas. Se trata de una característica de los sistemas familiares que es necesaria para construir nuevos proyectos de vida. Otra condición para lograr la adaptación es, la activación de su capacidad resiliente que se hace visible a través de las innumerables estrategias desplegadas parea lograr su sobrevivencia (López, 2005). Se entiende por resiliencia aquella capacidad que tiene la persona para hacer frente al dolor, experiencias difíciles 0 traumáticas (Wilches 2010). La capacidad de ser resiliente dependerá del grado de intensidad y duración o permanencia de las circunstancias adversas por las que se ha pasado (Becoña 2006).

Los servicios con los que cuentan las familias son: educación, seguridad, alumbrado, zonas recreativas, limpia y agua; de los servicios antes descritos, el de agua potable representa para todas las familias una muy mala calidad, debido a que el olor y color es desagradable. A continuación, se presenta la siguiente gráfica de acuerdo a los años que algunas familias llevan viviendo en el Fraccionamiento.

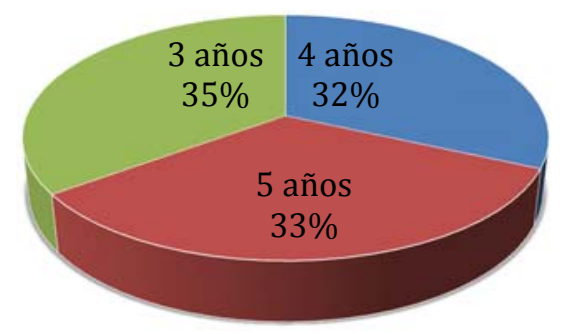

\section{Figura 1. Años viviendo en el Fraccionamiento.}

Fuente: Elaboración propia.

De las entrevistas realizadas el 35\% refleja que lleva 3 años viviendo en el Fraccionamiento, la casas que habitan las han adecuado a sus necesidades e integrantes de cada familia, así mismo algunos optaron por rentar o prestar sus inmuebles a interesados; realizan siembra de variedad de árboles ya que sus viviendas eran de solo tierra y pie de casa.

Sin duda, este proceso de adaptación de años sitúa a las familias a un proceso de reconstrucción individual y familiar en situación vulnerable y condiciones precarias o difíciles, tales como aquellas de su entorno como la mala calidad del agua por el tiempo que llevan viviendo en la zona, vivienda propia en el sentido que realmente sean propietarios de la vivienda, así como aquellas situaciones externas como la búsqueda de empleos que le queden cercano a su vivienda, seguridad, RUÍZ-RAMÓN M. G. 
DESPLAZAMIENTO FORZADO INTERNO COMO CONSECUENCIA DE UNA INUNDACIÓN EN LA ENTIDAD DE TABASCO, MÉXICO.

educación y alimentación. A pesar de todo ello las familias persisten en transformar y adaptar su vida cotidiana en aquello que pueda mejorar su condición de vida, en específico han ido en las adecuaciones realizadas a las viviendas (Briones, Audefroy \& Arévalo 2013).

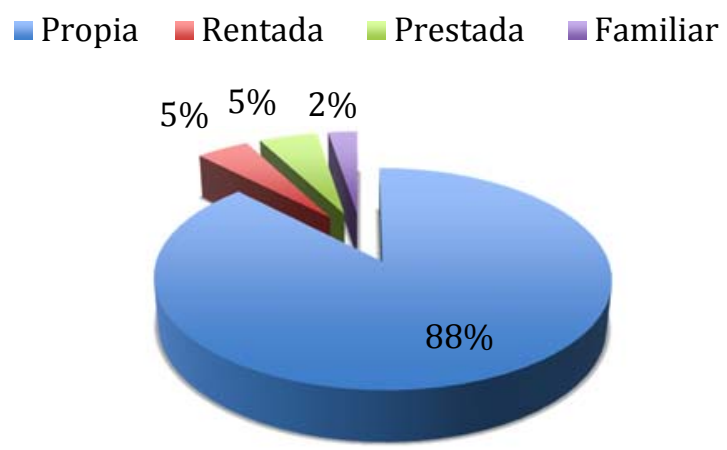

Figura 2. De la casa que habitan.

Fuente: Elaboración propia.

En la figura anterior, más del 50\% de los encuestados señalaron que es casa propia, aun cuando manifestaban que no tenían documentos; el 10\% señaló que es casa rentada o prestada por algún familiar directo o amigo de forma temporal, solo un $2 \%$ exteriorizó que la vivienda es de un familiar.

Las causas del desplazamiento de las familias al Fraccionamiento fueron a raíz de las inundaciones, estas familias si bien no son víctimas de lo acontecido, si se sitúan en un posicionamiento de vulnerabilidad y riesgo (Meier, 2007), ya que padecen de acumulación de los desechos en los hogares lo que propicia que en tiempos de lluvia el agua quede estancada, desencadenando encharcamientos y enfermedades como el dengue. En la siguiente figura, se muestra que los plásticos, desechos orgánicos y el papel son de los desechos que mayormente se genera en la comunidad, no existiendo una separación de ello y además en el momento en existe ausencia del servicio de limpia las familias acumulan o ponen en las esquinas de las calles los desechos. 
DESPLAZAMIENTO FORZADO INTERNO COMO CONSECUENCIA DE UNA INUNDACIÓN EN LA ENTIDAD DE TABASCO, MÉXICO.

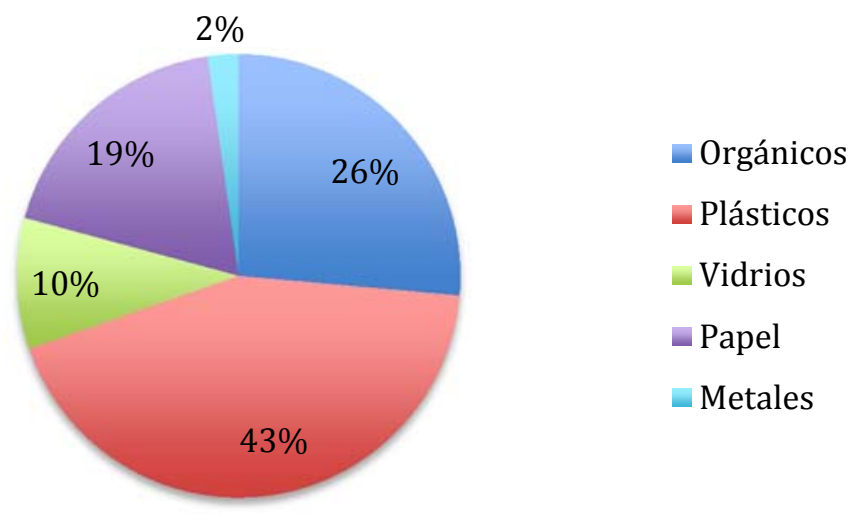

Figura 3. Desechos que se generan en el hogar.

Fuente: Elaboración propia.

Un aspecto a destacar es que si bien los desechos en su mayoría son plásticos como lo señala la gráfica 3 con un cuarenta y tres por ciento, en un veintiséis por ciento orgánicos (alimentos), y un diecinueve por ciento papel; estos residuos pueden considerarse de mayor aprovechamiento para su venta o en su caso procesos de reciclaje, composta; es decir, que el uso no sea de solo una ocasión sino reconvertir los desechos y originar mayores beneficios de forma particular, familiar, comunitaria. Así entonces se integra una solución duradera de las personas que antes estaban en situación de desplazamiento interno disfrutando de sus derechos humanos sin ser discriminados por esa condición; y [entre los medios para lograrlo] está [...]: La integración local sostenible en las zonas en que se hayan refugiado los desplazados internos [...] (Naciones Unidas, 2007).

Se concreta entonces un sistema de medios de vida sostenible en el quehacer de considerar diversos factores para un estado de bienestar y vida digna de la persona. Al respecto se entiende por medios de vida" o "medios de vida sostenibles" a aquellos activos tangibles e intangibles que contienen el acceso y disponibilidad a comida, ingresos y bienes; desde luego que incluyen a la gente y su capacidad para acceder y disponer a dichos medios de vida. Es medioambientalmente sostenible cuando se mantiene, incrementa y beneficia la reproducción de otros bienes, y es socialmente sostenible cuando permanece o se recupera después de un evento catastrófico y está disponible para futuras generaciones (Martínez, López, Álvarez \& Schmook 2016).

A pesar de ser una comunidad relativamente pequeña la generación de los desechos (Prats, et al., 2020) en la localidad ha generado una situación desagradable en el que las familias no se RUÍZ-RAMÓN M. G. 
DESPLAZAMIENTO FORZADO INTERNO COMO CONSECUENCIA DE UNA INUNDACIÓN EN LA ENTIDAD DE TABASCO, MÉXICO.

logran organizar para el uso y manejo de los mismos. Derivado de que el setenta y un por ciento de los encuestados señalan que acumulan los desechos cuando no pasa el camión recolector y un veintiún por ciento la ponen en las esquinas, propicia que en ocasiones los animales que andan en la calle como perros y gatos rompan las bolsas de basura ocasionando así molestias entre los habitantes. Asimismo, todavía un ocho por ciento de la población quema los desechos, siendo esto un mecanismo pronto para deshacerse de los mismos. Por lo cual se reitera que no existe un proceso de educación y cultura ambiental, en el que las personas puedan sacar provechos de los desechos y no generarlo como basura.

Un caso de éxito a nivel nacional en atención a procesos de educación y cultura ambiental de forma integral vinculada con otras formas de vida de la comunidad es el caso de Cherán, Michoacán, en el que el proceso de movilización y de reconstrucción social impulsó un amplio abanico de acciones para la transformación de conflictos que abarca muchas de sus dimensiones. Destacan el impulso a actividades culturales y deportivas para jóvenes y niños, la separación y el reciclaje de la basura y la inclusión de la ciudadanía en las tareas de seguridad pública (Gasparello 2018).

La situación de organización y procesos de adaptación a ciertas circunstancias a pesar de los años de habitar en el fraccionamiento hace que puedan proliferar conflictos entre los habitantes de dicha comunidad.

Para ello, los habitantes están organizados de diversas formas, si bien hay ausencia de organización para las situaciones que afectan el bien común como los desechos, existe también la forma de organización religiosa en la que pondera mayor organización y comunicación, un ejemplo de ello es la recaudación de fondos con el objetivo de mejorar la infraestructura de sus iglesias; los programas sociales representan para algunas familias otro tipo de organización e ingreso.

Algo muy importante a destacar de los resultados es, que a pesar de que se carece de organización comunitaria, las personas están dispuestas a colaborar en acciones que les pueda beneficiar con el interés de crear y/o recuperar espacios verdes o de esparcimiento.

Es preciso destacar la disposición de algunas familias para el manejo y uso de los desechos, organización y planeación de estrategias en y para la comunidad. Este proceso, requiere ser visto desde un ámbito educativo el cual versa como primera instancia en el ámbito ambiental o RUÍZ-RAMÓN M. G. 
DESPLAZAMIENTO FORZADO INTERNO COMO CONSECUENCIA DE UNA INUNDACIÓN EN LA ENTIDAD DE TABASCO, MÉXICO.

ecológico basado en la formación e instrucción principalmente de los jóvenes, gestores, líderes, para la gestión, planificación e inclusión de valores en una relación responsable con la naturaleza; en segunda instancia el ámbito social, en la cual predomina el impulso de la transformación estructural de la comunidad; así que llevado a efecto de forma progresiva estas dos vertientes se estará trabajando en un proceso educativo ambiental que impacte como agente de cambio en la realidad social de las personas que habitan el Fraccionamiento.

Finalmente, la atención a la problemática de las personas que fueron desplazadas de forma interna debe ser interpretada de forma transversal, holística e integral; que permita la colaboración y cooperación a través de acciones o políticas públicas encaminadas a la acción participación y toma de decisiones de las personas que ahí habitan, así como considerar su contexto, necesidades e intereses de la comunidad.

\section{CONCLUSIONES}

- Es necesario que la aplicación de estrategias de adaptación, junto con la implementación de una buena gestión de riesgos de catástrofes, puede reducir, adecuar o manejar la vulnerabilidad frente al cambio climático, requiriendo que los diversos actores trabajen en distintos ámbitos, realicen un esfuerzo colectivo y traspasen los límites sectoriales.

- Las familias afectadas de las colonias descritas en el trabajo, tuvieron un proceso complejo de zozobra y arraigo a sus viviendas en "zona vulnerable" en principio como refugiados en albergues, algunas otras familias optaron por el éxodo a otras entidades o refugiarse en casa de familiares.

- El desplazamiento interno que vivieron las familias las orillo a adaptarse a nuevas formas de vida, donde ellos fueron los que se adaptaron a la zona en que habitan, y no el gobierno se adaptó a las necesidades de las familias.

- La infraestructura de la zona en que fueron desplazados no contaba con algunos servicios básicos y zonas de recreación, a lo que las familias decidieron reforestar áreas verdes y sembrar variedad de árboles en algunas casas, con la finalidad de obtener algunos frutos y obtención de sombra para sosegar el calor.

- Los desechos que se generan en la comunidad no tienen un tratamiento adecuado y el servicio de limpia algunas veces es ausente lo que propicia el acumulamiento y generación de desechos en hogares y calles de la comunidad, aunado a esto se propicia focos de infección en la zona. 
DESPLAZAMIENTO FORZADO INTERNO COMO CONSECUENCIA DE UNA INUNDACIÓN EN LA ENTIDAD DE TABASCO, MÉXICO.

- Algunos habitantes tienen el interés de participar en actividades de reforestación, manejo y uso de desechos, composta, campañas de limpieza y saneamiento, por lo que después de este diagnóstico se trabaja para la implementación de un plan de trabajo inter y multidisciplinario, con el apoyo de instituciones educativas, profesores y estudiantes.

\section{REFERENCIAS BIBLIOGRÁFICAS}

Becoña, E. (2006). Resiliencia: definición, características y utilidad del concepto. Revista de Psicopatología y Psicología Clínica, 11(3), 125-146.

Briones, F., Audefroy, J., Arévalo, M.L. (2013). ¿Reubicados o desplazados? Impactos sociales en la Ciudad Rural de Juan de Grijalva, Chiapas. El desplazamiento interno forzado en México. Un acercamiento para su reflexión y análisis, México, CIESAS, Senado de la República, El Colegio de Sonora.

Briones, G. (2001). Métodos y técnicas de investigación para las ciencias sociales. México: Trillas.

Comisión Mexicana de Defensa y Promoción de los Derechos Humanos (CMDPDH). (2019). Desplazamiento interno forzado en México. México.

Comisión Nacional de los Derechos Humanos (CNDH). (2016). Informe Especial sobre Desplazamiento Forzado Interno (DFI) en México. México.

Garza, A. (2013). Manual de técnicas de investigación para estudiantes de ciencias sociales y humanidades, México, Colegio de México.

Gasparello, G. (2018). Análisis del conflicto y de la violencia en Cherán, Michoacán. Relaciones estudios de historia y sociedad, 39(55).

Hernández, C. (2016). Neoextractivismo: tendencia del desplazamiento interno forzado. Revista mensual de la Comisión de Derechos Humanos del Distrito Federal, Dfensor, 4(XIV), abril. 20-25.

Jaimes, P. (2014). Desplazamiento forzado y derechos humanos. Tesis para la obtención de grado de Doctor. Universidad de Granada.

López, Olga L. (2005). La resiliencia de las familias afectadas por el desplazamiento forzado en Colombia, Perspectivas sociales, 7(2), 1-28

Martínez, G., López, M. S., Álvarez, G. y Schmook, B. (2017). Desastres, desplazamiento interno y migración labora en la Sierra de Chiapas, Papeles de población, 22(87).

RUÍZ-RAMÓN M. G.

SEPTIEMBRE-DICIEMBRE 2020. Año 26, Número 76. Págs. 338-351 
DESPLAZAMIENTO FORZADO INTERNO COMO CONSECUENCIA DE UNA INUNDACIÓN EN LA ENTIDAD DE TABASCO, MÉXICO.

Meier, R. (2007). “¿Por qué son víctimas las personas desplazadas?” Boletín Hechos del Calle, año 3. Bogotá: PNUD.

Naciones Unidas. (2007). Cuando termina el desplazamiento interno. Marco de soluciones duraderas, junio de 2007, Institución Brookings-Universidad de Berna, proyecto sobre desplazamiento interno.

Naciones Unidas. (1998). Principios Rectores de los Desplazamientos Internos. Nueva York. Recuperado de https://www.acnur.org/prot/prot_despl/5bff2c864/principios-rectores-de-losdesplazamientos-internos.html

Prats, G., Álvarez García, Y., Hernández, F. \& Zamora, D. (2020). Environmental Taxes. Its Influence on Solid Waste in Mexico. Journal Of Environmental Management And Tourism, 11(3), 755-762. doi:10.14505//jemt.v11. 3(43).29

Prieur, M. (2009). La necesidad de un estatuto internacional de los desplazados ambientales, ponencia en Congreso Jurídico Internacional, Globalización, riesgo y medio ambiente, 3 al 5 marzo 2010, Universidad de Granada.

Quintero, M. L. (2005). Características fundamentales de las políticas ambientales: el riesgo y la incertidumbre, Aportes, mayo-agosto, año/Vol. X, número 029, Benemérita Universidad de Puebla. México.

Salazar, L. M. (2014). Modalidades del desplazamiento interno forzado en México. Iztapalapa, Revista de Ciencias Sociales y Humanidades, 76(35), enero-junio.

Silva Hernández, Francisca. (2019). Principio de prevención y precautorio en materia ambiental. Revista Jurídica Derecho, 8(11), 92-106.

Silva, F. y Martínez, G. (2019). La justicia alternativa como derecho humano. JURÍDICASCUC, 15(1), 263-284. https://doi.org/10.17981/juridcuc.15.1.2019.10

Stavropoulou, M. (2008). ¿Un mar de definiciones? Revista: migraciones forzadas, Universidad de OXFORD, 31.

Wilches, I. (2010). Lo que hemos aprendido sobre la atención a mujeres víctimas de violencia sexual en el conflicto armado colombiano. Revista Universidad de Los Andes, 36, 86-94. doi: $10.7440 /$ res36.2010.08 